\title{
Erratum to: Formal Methods for Distributed System Development
}

\author{
Tommaso Bolognesi ${ }^{1}$ and Diego Latella ${ }^{2}$ \\ 1 Consiglio Nazionale delle Ricerche (CNR), \\ Istituto di Elaborazione dell'Informazione (IEI), Italy \\ 2 Consiglio Nazionale delle Ricerche (CNR), Istituto CNUCE, Italy
}

\section{Erratum to:}

\section{Tommaso Bolognesi and Diego Latella (Eds.)}

Formal Methods for Distributed System Development

DOI: $10.1007 / 978-0-387-35533-7$

The book was inadvertently published with an incorrect name of the copyright holder. The name of the copyright holder for this book is: (c) IFIP International Federation for Information Processing. The book has been updated with the changes.

The updated original online version for this book can be found at DOI: $10.1007 / 978-0-387-35533-7$

Tommaso Bolognesi and Diego Latella (Eds.), Formal Methods for Distributed System Development. 